\title{
ROTATING SKYRMIONS OF THE $(2+1)$-DIMENSIONAL SKYRME GAUGE MODEL WITH A CHERN-SIMONS TERM
}

\author{
Loginov A.Yu. \\ Tomsk Polytechnic University, Russia \\ E-mail: aloginov@tpu
}

The $(2+1)$-dimensional Skyrme gauge model [1] with a Chern-Simons term [2] is considered. The presence of the Chern-Simons term makes possible the existence of two dimensional skyrmions in this model that carry magnetic flux and have electric charge and nonzero angular momentum [3]. It is shown that the model also admits the existence of two dimensional skyrmions with nonzero phase frequency of rotation. Due to the nontrivial topological properties of the model, the magnetic flux, electric charge, and the angular momentum of a two dimensional rotating skyrmion turn out to be related to each other. Analytic and numerical investigations of the properties of rotating two dimensional skyrmions are carried out.

1. J.Gladikowski, B.M.A.G.Piette, B.J.Schroers // Phys. Rev. D. 1996. V.53. P.844.

2. R.Jackiw, S.Templeton // Phys. Rev. D. 1981. V.23. P.2291.

3. S.K.Paul, A.Khare // Phys. Lett. B. 1986. V.174. P.420. 